\title{
Some features of semiclassical chiral transport in rotating frames
}

\author{
Ömer F. Dayi and Eda Kilinçarslan \\ Physics Engineering Department, Faculty of Science and Letters, Istanbul Technical University, \\ TR-34469, Maslak-Istanbul, Turkey
}

(Received 13 June 2019; published 13 August 2019)

\begin{abstract}
Semiclassical chiral kinetic theories in the presence of electromagnetic fields as well as vorticity can be constructed by means of some different relativistic or nonrelativistic approaches. To cover the noninertial features of rotating frames one can start from the modified quantum kinetic equation of Wigner function in Minkowski spacetime. It provides a relativistic chiral transport equation whose nonrelativistic limit yields a consistent three-dimensional kinetic theory which does not depend explicitly on spatial coordinates. Recently a chiral transport equation in curved spacetime has been proposed and its nonrelativistic limit in rotating coordinates was considered in the absence of electromagnetic fields. We show that the modified theory can be extended to curved spacetime. The related particle current density and chiral transport equation for an inertial observer in the rotating frame are derived. A novel three-dimensional chiral kinetic transport equation is established by inspecting the nonrelativistic limit of the curved spacetime approach in the rotating frame for a comoving observer in the presence of electromagnetic fields. It explicitly depends on spatial coordinates. We prove that it is consistent with the chiral anomaly, chiral magnetic and vortical effects.
\end{abstract}

DOI: 10.1103/PhysRevD.100.045012

\section{INTRODUCTION}

In the presence of external electromagnetic fields the charged, massless Dirac particles which can be right- or left-handed, exhibit unusual features like the chiral magnetic effect [1-3] and the chiral separation effect [4,5]. These manifest themselves in heavy-ion collisions [1,2]. There are also some similar phenomena due to rotation of coordinate frame which affect dynamical behavior of chiral particles: The chiral vortical effect [6-10] and the local polarization effect [11-13].

Dynamical features of chiral particles have been investigated mostly within the semiclassical kinetic theories which offer an intuitive understanding of their collective phenomena. Kinetic theories can be studied either in a manifestly covariant way in Minkowski spacetime or within the nonrelativistic approach where the physical content is apparent. Three-dimensional (3D) semiclassical chiral transport theories based on [14,15], are usually inspected by making allowance for only the external electromagnetic fields or the rotation of coordinate frame. The first 3D semiclassical kinetic theory of chiral particles which takes into account the external electromagnetic fields as well as the rotation of the coordinate frame and

Published by the American Physical Society under the terms of the Creative Commons Attribution 4.0 International license. Further distribution of this work must maintain attribution to the author(s) and the published article's title, journal citation, and DOI. Funded by SCOAP . consistent with the chiral anomaly was constructed in [16]. It generates the chiral anomalous effects correctly as it should be.

A relativistic chiral kinetic theory (CKT) can be defined [17-19] starting from the quantum kinetic equation (QKE) obeyed by the Wigner function [20,21]. However, it does not take into account all noninertial effects like the Coriolis force. To overcome this shortcoming QKE was modified by means of some frame dependent terms in [22]. To have better insights into the transport properties of particles, it is convenient to consider the 3D CKT generated by the relativistic chiral transport equation (CTE). However, this limit is challenging, the nonrelativistic CKT should be consistent with chiral anomaly and anomalous currents. The modified approach [22] gives rise to a consistent 3D CKT which does not explicitly depend on the spatial coordinates $\boldsymbol{x}$, in the presence of both electrodynamic fields and vorticity. It is preferable to work with a CTE which does not explicitly depend on the local positions of particles but global features like the angular velocity $\boldsymbol{\omega}$ of the rotating frame. Nonetheless, explicit rotation velocity dependence is pertinent to 3D transport equations. In fact, the 3D CTE established in [16] depends explicitly on $\boldsymbol{u}=\boldsymbol{x} \times \boldsymbol{\omega}$. This dependence is crucial in obtaining the continuity relation: If the rotation velocity dependent terms are suspended, there will appear some undesired terms in the Liouville equation.

Relativistic chiral kinetic theories which we deal with are the ones provided by the quantum kinetic equation without referring explicitly to the equilibrium distribution 
functions. In the approach of $[23,24]$ the solution of quantum kinetic equation derived in [13] was employed to define a CKT. Yet another methods of furnishing chiral transport equations were presented in $[25,26]$.

Recently CKT was studied in curved spacetime where the 3D limit in rotating coordinates has been exposed only for vanishing electromagnetic fields [27]. Considering QKE in curved spacetime [28] effectively means to modify it in a frame dependent manner. In fact the Coriolis force has been acquired by choosing the metric adequate to deal with rotating coordinates [27]. The nonrelativistic limit in the absence of electromagnetic fields was discussed for two different choices of four-velocities corresponding to inertial and rotating observers. For the latter choice the CTE which they obtained is the same with the one derived in [16] at $\boldsymbol{x} \approx 0$. We would like to understand how the formulations established in [16] and [22] are related to the curved space formulation of chiral particles in rotating coordinates. We will first show that the modified QKE approach can be extended to curved spacetime. We study it in rotating coordinates by choosing adequately the metric tensor. The modification is relevant only for an inertial observer as far as the centrifugal force is ignored. We calculate the particle current density for an inertial observer in terms of the equilibrium distribution function resulting from two different choices of fluid fourvelocities. Then the CKE in the absence of electromagnetic fields is obtained. The phase space measure and first time derivatives of phase space variables which we acquire, differ from the ones presented in [27]. In fact, CKT which we acquire is partially in accord with the one established in [16]. We then study the 3D limit of four-dimensional CTE for a rotating observer in the presence of electromagnetic fields. The CKT which follows possesses $\boldsymbol{x}$ dependent terms and resembles the one established in [16]. We show that it is consistent with the chiral anomaly, the chiral vortical and magnetic effects. Hence it constitutes a new 3D CTE.

In Sec. II we briefly review the QKE and the existing modifications which take into account noninertial properties of rotating coordinates. The modified kinetic equation in curved space is presented in Sec. III. The particle current density and the resulting 3D CKT are obtained for an inertial observer. Section IV is devoted to CKT for a rotating observer in the presence of external electromagnetic fields. We obtain a novel 3D CKT which gives rise to the continuity equation and anomalous chiral effects correctly. In the last section the results acquired and future directions are discussed.

\section{WIGNER FUNCTION FORMALISMS}

Chiral theories which we consider are based on the quantum kinetic equation

$$
\gamma^{\mu}\left(p_{\mu}+\frac{i \hbar}{2} \mathfrak{D}_{\mu}^{(I)}\right) W(x, p)=0
$$

$\left(x_{\mu}, p_{\mu}\right)$ are the eight-dimensional phase space variables. The superscript $I=(O, M, C)$, standing for original, modified and curved, indicates the different choices of derivative terms. As it will explicitly be given shortly, their differences lie in how they treat the inertial properties of coordinate frame. $W(x, p)$ is the Wigner function for spin- $1 / 2$ fermions which can be decomposed in terms of the Clifford algebra generators whose coefficients are the scalar, pseudoscalar, vector, axial-vector and tensor fields. We are interested in the chiral vector fields given by the vector and axial-vector field components $\mathcal{V}_{\mu}$ and $\mathcal{A}_{\mu}$ as

$$
\mathcal{J}_{\chi}^{\mu}=\frac{1}{2}\left(\mathcal{V}^{\mu}+\chi \mathcal{A}^{\mu}\right)
$$

$\chi= \pm 1$, indicates the right- and left-handed fermions. We take into consideration only the right-handed chiral vector field to expose our results, $\mathcal{J}^{\mu} \equiv \mathcal{J}_{1}^{\mu}$. The equations which they obey decouple from the other components, leading to

$$
\begin{aligned}
p_{\mu} \mathcal{J}^{\mu} & =0, \\
\hbar \epsilon^{\mu \nu \alpha \rho} \mathfrak{D}_{\alpha}^{(I)} \mathcal{J}_{\rho} & =-2\left(p^{\mu} \mathcal{J}^{\nu}-p^{\nu} \mathcal{J}^{\mu}\right), \\
\mathfrak{D}_{\mu}^{(I)} \mathcal{J}^{\mu} & =0 .
\end{aligned}
$$

The original QKE $[20,21]$ was derived starting from the Dirac equation in Minkowski spacetime yielding the derivative terms

$$
\mathfrak{D}_{\mu}^{(O)} \equiv \nabla_{\mu}=\partial_{\mu}-F_{\mu \nu} \partial_{p}^{\nu}
$$

The derivatives with respect to the phase space variables denoted $\partial_{\mu} \equiv \partial / \partial x^{\mu}, \partial_{p}^{\mu} \equiv \partial / \partial p_{\mu}$. We set $c=k=1$ as well as $Q=1$ which is the electric charge of chiral particle coupled to the external electromagnetic fields described by $F_{\mu \nu}$. For the sake of simplicity we deal with the electromagnetic field strength satisfying $\partial_{\rho} F_{\mu \nu}=0$.

In spite of the fact that the original QKE is covariant, it does not explicitly depend on the inertial properties of reference frame. The fluid vorticity or equivalently the angular velocity of the frame appears in its solution [13]. Therefore, the CTE designated by (5) does not lead to the Coriolis force. To surmount this disadvantage in [22] the derivative terms of the original QKE is modified by means of the four-velocity $n^{\mu}$ of the frame satisfying $n_{\mu} n^{\mu}=1$, as

$$
\mathfrak{D}_{\mu}^{(M)} \equiv \tilde{\nabla}_{\mu}^{(n)}=\nabla_{\mu}+\left[\partial_{\nu} n^{\alpha} p_{\alpha} n_{\mu}-\partial_{\mu} n^{\alpha} p_{\alpha} n_{\nu}\right] \partial_{p}^{\nu} .
$$

We introduced the modification guided by the circulation tensor which provides the noninertial properties of fluids. This approach revealed to be essential in obtaining a formulation of 3D CTE which is not explicitly dependent of the spatial coordinates $\boldsymbol{x}$. In fact, it is the unique $\boldsymbol{x}$ independent 3D CKT consistent with the chiral anomaly 
when both the external electromagnetic fields and fluid vorticity are present. Hence, as far as these properties are considered the modification terms seem to be unique. This formalism possesses some similarities with the effective field theory approach [8] but it is not possible to introduce a gauge field which generates the proposed modification of QKE.

On the other hand frame dependent terms can also be incorporated in the original QKE by extending it to curved spacetime. This has been achieved by means of "horizontal lift of the derivative operator in the cotangent bundle" $[27,28]$ yielding

$$
\mathfrak{D}_{\mu}^{(C)} \equiv \Delta_{\mu}=\partial_{\mu}+\left[\Gamma_{\mu \nu}^{\lambda} p_{\lambda}-F_{\mu \nu}\right] \partial_{p}^{\nu} .
$$

$\Gamma_{\mu \nu}^{\lambda}$ denote the Christoffel symbols. We suppressed spin connection because it does not show up in (2)-(4).

We are concerned with the semiclassical approximation where the Wigner function is expanded in Planck constant and the terms up to the first order are retained. In course of obtaining CKT one first solves (2) and (3). For $\nabla_{\mu}$, they were solved in [17-19]. As it has been demonstrated in [27], this solution can be extended to the curved spacetime by substituting $\nabla_{\mu}$ with $\Delta_{\mu}$, giving

$\mathcal{J}^{\mu}=p^{\mu} f \delta\left(p^{2}\right)+\hbar \tilde{F}^{\mu \nu} p_{\nu} f \delta^{\prime}\left(p^{2}\right)+\hbar S_{(n)}^{\mu \nu}\left(\Delta_{\nu} f\right) \delta\left(p^{2}\right)$,

where $\delta^{\prime}\left(p^{2}\right)=d \delta\left(p^{2}\right) / d p^{2}$. One also expands the distribution function in $\hbar$ and retain the first order: $f \equiv f^{0}+\hbar f^{1}$. The spin tensor

$$
S_{(n)}^{\mu \nu}=\frac{\epsilon^{\mu \nu \rho \sigma} p_{\rho} n_{\sigma}}{2 n \cdot p},
$$

and the dual electromagnetic field strength $\tilde{F}^{\mu \nu}=\epsilon^{\mu \nu \alpha \rho} F_{\alpha \rho} / 2$, are introduced. By making use of (8) in the remaining Eq. (4) one attains the relativistic CKT in curved spacetime:

$\delta\left(p^{2}+\frac{\hbar n_{\alpha} \tilde{F}^{\alpha \beta} p_{\beta}}{n \cdot p}\right)\left[p \cdot \Delta+\frac{\hbar n_{\mu} \tilde{F}^{\mu \nu} \Delta_{\nu}}{n \cdot p}+\hbar \Delta_{\mu} S_{(n)}^{\mu \nu} \Delta_{\nu}\right] f=0$.

We would like to examine the behavior of massless Dirac particles in rotating coordinates. Metric components of the coordinate frame which rotates with the constant angular velocity $\boldsymbol{\omega}$ were given in $[29,30]$ as

$$
g_{00}=1-\boldsymbol{u}^{2}, \quad g_{0 i}=u^{i}, \quad g_{i j}=-\delta_{i j} .
$$

We deal with nonrelativistic rotations, thus we let the rotation velocity $u^{i}=\epsilon^{i j k} x^{j} \omega^{k}$ be much smaller than the speed of light: $|\boldsymbol{u}| \ll 1 . \epsilon^{i j k}$ is totally antisymmetric and $\epsilon^{123}=1$.
The related Christoffel symbols can be seen to be

$$
\Gamma_{0 i}^{j}=\Gamma_{i 0}^{j}=\epsilon^{i j k} \omega^{k}, \quad \Gamma_{00}^{i}=\epsilon^{i j k} u^{j} \omega^{k}, \quad \Gamma_{\mu \nu}^{0}=\Gamma_{j k}^{i}=0 .
$$

It yields a vanishing Riemann tensor.

Following [27] we consider two different observers: The inertial observer with the four-velocity

$$
u_{\mu}=(1,0,0,0), \quad u^{\mu}=\left(1, u^{i}\right),
$$

and the rotating observer with the four-velocity

$$
v^{\mu}=\frac{1}{\sqrt{g_{00}}} \delta_{0}^{\mu}, \quad v_{\mu}=\left(\sqrt{g_{00}}, \frac{1}{\sqrt{g_{00}}} u^{i}\right) .
$$

The 3D CKT established in [16] embraces the Coriolis force as well as the centrifugal force. However, when one studies the 3D theories generated by the 4D CKTs the centrifugal terms are ignored. Also here we do not take into account those effects which means that $O\left(\boldsymbol{u}^{2}\right)$ terms and their derivatives with respect to spatial coordinates assumed to be vanishing. In accord with this assumption we adopt the approximation

$$
O\left(u^{i} u^{j}\right) \approx 0, \quad O\left(u^{i} \omega^{j}\right) \approx 0 .
$$

It is worth mentioning that this approximation is consistent with the fact that $|\boldsymbol{u}| \ll 1$.

\section{MODIFIED KINETIC EQUATION IN CURVED SPACETIME}

The modified theory can be extended to the curved spacetime by substituting $\mathfrak{D}_{\mu}^{(I)}$ in (1) with

$$
\Delta_{(n) \mu}=\partial_{\mu}+\left[\Gamma_{\mu \nu}^{\lambda} p_{\lambda}-F_{\mu \nu}+\partial_{\mu} n^{\alpha} p_{\alpha} n_{\nu}-\partial_{\nu} n^{\alpha} p_{\alpha} n_{\mu}\right] \partial_{p}^{\nu} .
$$

The signs of modification terms are altered with respect to (6) as it is convenient for considering $p_{\mu}$ as the momentum variables. The semiclassical solution of (2) and (3) can be attained as

$$
\begin{aligned}
\mathcal{J}_{(n)}^{\mu}= & \left(1-\frac{\hbar}{n \cdot p} S_{(n)}^{\sigma \rho} g_{\rho \nu}\left(\partial_{\sigma} n^{\nu}\right)\right) p^{\mu} f \delta\left(p^{2}\right) \\
& +\hbar \tilde{F}^{\mu \nu} p_{\nu} f \delta^{\prime}\left(p^{2}\right)-\hbar \epsilon^{\mu \nu \alpha \rho} p_{\nu}\left(\partial_{\alpha} n^{\beta}\right) p_{\beta} n_{\rho} f \delta^{\prime}\left(p^{2}\right) \\
& +\hbar S_{(n)}^{\mu \nu}\left(\Delta_{(n) \nu} f^{0}\right) \delta\left(p^{2}\right) .
\end{aligned}
$$

We deal with the semiclassical approximation, so that in (13) the first order part of the distribution function $f^{1}$, is arbitrary. In fact, we used this freedom to write the second term in accord with the Minkowski spacetime formulation [22]. It is worth noting that to determine the distribution 
function completely one should solve the remaining equation (4).

We first would like to discuss 3D currents resulting from (13) in rotating coordinates by choosing $n_{\mu}$ appropriately. Because of ignoring the centrifugal effects we deal with $\boldsymbol{u}$ satisfying (11). Then, the unique possibility is to choose $n_{\mu}=u_{\mu}$, because for $n_{\mu}=v_{\mu}$ the modification terms in (12) vanish.

The zeroth component of (13) yields particle number density and the spatial components are used to define the chiral particle current density as

$$
j_{(u)}^{i}=\int \frac{d^{4} p}{4 \pi^{3} \hbar^{3}} \mathcal{J}_{(u)}^{i}
$$

We would like to emphasize the fact that terms which are at most linear in $\hbar$ are kept, obviously up to the $\hbar^{-3}$ factor which is due to the definition of momentum space volume. Its partial integration reads

$$
\begin{aligned}
j_{(u)}^{\mu}= & \int \frac{d^{4} p}{4 \pi^{3} \hbar^{3}}\left[p^{\mu} f-\hbar \partial_{p}^{0}\left(\tilde{F}^{\mu \nu} p_{\nu} f / 2 p_{0}\right)\right. \\
& +\hbar \epsilon^{\mu \nu \alpha \rho} \partial_{p}^{0}\left(p_{\nu}\left(\partial_{\alpha} u^{\beta}\right) p_{\beta} u_{\rho} f / 2 p_{0}\right) \\
& \left.-\frac{\hbar}{u \cdot p} p^{\mu} S_{(u)}^{\lambda \rho} g_{\rho \nu}\left(\partial_{\lambda} u^{\nu}\right) f^{0}+\hbar S_{(u)}^{\mu \nu}\left(\Delta_{(u) \nu} f^{0}\right)\right] \delta\left(p^{2}\right) .
\end{aligned}
$$

We adopt the equilibrium distribution function for rotating coordinates as it is given in [27,31]:

$$
f_{e q}(x, p)=\left[1+e^{\left(p \cdot U-\mu+\frac{\hbar}{2} S_{(n)}^{\mu \nu} \partial_{\mu} U_{\nu}\right) / T}\right]^{-1} .
$$

$U_{\mu}$ is the four-velocity of fluid and $n_{\mu}=u_{\mu}$.

One can first perform the $p_{0}$ integral by solving the mass-shell condition $p^{2}=0$ as

$$
p_{0}= \pm|\boldsymbol{p}|-p_{i} u^{i}
$$

where $|\boldsymbol{p}|=\sqrt{p_{i} p_{i}}$. Then we write

$$
\begin{aligned}
\delta\left(p^{2}\right)= & \frac{\theta\left(p_{0}\right) \delta\left(p_{0}-|\boldsymbol{p}|+p_{i} u^{i}\right)}{2|\boldsymbol{p}|} \\
& +\frac{\theta\left(-p_{0}\right) \delta\left(p_{0}+|\boldsymbol{p}|+p_{i} u^{i}\right)}{2|\boldsymbol{p}|} .
\end{aligned}
$$

We only display the positive part explicitly. After integrating over $p_{0}$, in the vicinity of $\boldsymbol{x} \approx 0$ the current density can be written as

$$
\begin{aligned}
j_{(u)}^{i}= & \int \frac{d^{3} p}{(2 \pi \hbar)^{3}}\left[-p_{i}+\frac{\hbar}{2|\boldsymbol{p}|} \omega^{i}-\frac{3 \hbar}{2|\boldsymbol{p}|^{3}} \omega^{j} p_{j} p_{i}\right. \\
& +\frac{\hbar}{2|\boldsymbol{p}|} \epsilon^{i j k} F_{j k} \frac{\partial}{\partial|\boldsymbol{p}|}+\frac{\hbar}{2|\boldsymbol{p}|^{3}} \epsilon^{i j k} p_{j} F_{0 k} \\
& \left.+\frac{\hbar}{2|\boldsymbol{p}|^{2}} \epsilon^{i j k} p_{j} \frac{\partial}{\partial x_{k}}\right]\left.f_{e q}\right|_{p_{0}=|\boldsymbol{p}|-p_{i} u^{i}} .
\end{aligned}
$$

Observe that $p_{i} / 2|\boldsymbol{p}|^{3}$ is the Berry curvature.

There are two possibilities of choosing the fluid fourvelocity in the equilibrium distribution (14). For $U_{\mu}=u_{\mu}$, contributions arising from the second and third terms in (15) cancel each other, so that the chiral vortical effect is not generated. (15) only leads to the magnetic current

$$
\boldsymbol{j}_{(u)}^{B}=\frac{\mu}{4 \pi^{2} \hbar^{2}} \boldsymbol{B},
$$

where $\frac{1}{2} \epsilon^{i j k} F_{j k}=-B^{i}$. It provides the chiral magnetic effect. The other possibility is to set $U_{\mu}=v_{\mu}$, which yields $\boldsymbol{j}_{(u)}=\boldsymbol{j}_{(u)}^{B}+\boldsymbol{j}_{(u)}^{\omega}$, where

$$
\boldsymbol{j}_{(u)}^{\omega}=\left(\frac{\mu^{2}}{2}+\frac{T^{2} \pi^{2}}{6}\right) \frac{\omega}{2 \pi^{2} \hbar^{2}},
$$

describes the chiral vortical effect correctly. Obviously, to work out the momentum integrals both particle and antiparticle contributions should be taken into account. Although angular velocity dependence of the chiral current obtained in [27] differs from (15), the results regarding the chiral vortical effect are consistent.

Let us switch off the external electromagnetic fields and plug (13) into the remaining Eq. (4). For the sake of simplicity we set terms which are second order in angular velocity into zero and work in the vicinity of $\boldsymbol{x} \approx 0$. After integrating over $p_{0}$ we obtain

$$
\left(\sqrt{\kappa} \frac{\partial}{\partial t}+\sqrt{\kappa} \dot{x}^{i} \frac{\partial}{\partial x^{i}}+\sqrt{\kappa} \dot{p}_{i} \frac{\partial}{\partial p_{i}}\right) f(t, \boldsymbol{x}, \boldsymbol{p})=0,
$$

where $f(t, \boldsymbol{x}, \boldsymbol{p}) \equiv f_{e q}\left(x, p_{i}, p_{0}=|\boldsymbol{p}|-p_{i} u^{i}\right)$. The phase space measure and the first time derivatives of phase space variables are

$$
\begin{gathered}
\sqrt{\kappa}=1+\frac{p_{i} \omega^{i}}{|\boldsymbol{p}|^{2}}, \\
\sqrt{\kappa} \dot{x}^{i}=-\mathrm{v}^{i}-\frac{\hbar \hat{p}_{j} \omega^{j} \hat{p}_{i}}{|\boldsymbol{p}|}, \\
\sqrt{\kappa} \dot{p}_{i}=2|\boldsymbol{p}| \epsilon^{i j k} \mathrm{v}^{j} \omega^{k} .
\end{gathered}
$$

We introduced the "canonical velocity" 


$$
\mathrm{v}^{i}=\hat{p}_{i}-\frac{\hbar \omega^{i}}{2|\boldsymbol{p}|}+\frac{\hbar \hat{p}_{j} \omega^{j} \hat{p}_{i}}{2|\boldsymbol{p}|} .
$$

(17) and (19) are in accord with the CKT derived in [16]. Let us compare (16)-(19) with the CKT obtained for inertial fluid in [27] where phase space measure is 1 and $\dot{x}^{i}=-\hat{p}_{i}+u^{i}, \dot{p}_{i}=\epsilon^{i j k} p_{j} \omega^{k}$. First of all, some versions of CKT are related by phase space coordinate transformations [32], seemingly this is not the case for the formalisms which we compare. In the latter approach phase space measure and $\dot{\boldsymbol{x}}$ at $\boldsymbol{x} \approx 0$, do not possess angular velocity dependence and in contrary to (19) Coriolis force with the factor of 2 is generated if one does not suppress the $\boldsymbol{x}$ dependence. In fact, the latter formalism is classical but the one derived here possesses quantum corrections. This fact will be important especially when one considers collisions. Moreover, in Minkowski spacetime the modification of QKE is necessary to obtain a Coriolis like term and a satisfactory $\boldsymbol{x}$ dependent 3D CKT in the presence of electromagnetic fields.

\section{3D CKT IN THE PRESENCE OF ELECTROMAGNETIC FIELDS}

In this section we let the particle 4-velocity be $U_{\mu}=v_{\mu}$ and consider the 4D CKT in the comoving frame $n_{\mu}=v_{\mu}$, provided by (9) as

$\delta\left(p^{2}+\frac{\hbar v_{\alpha} \tilde{F}^{\alpha \beta} p_{\beta}}{v \cdot p}\right)\left\{p \cdot \Delta+\frac{\hbar v_{\mu} \tilde{F}^{\mu \nu} \Delta_{\nu}}{v \cdot p}+\hbar \Delta_{\mu} S_{(v)}^{\mu \nu} \Delta_{\nu}\right\} f=0$.

In [27] this transport equation was studied in the absence of electromagnetic fields. They showed that working with $p^{\mu}=g^{\mu \nu} p_{\nu}$, suits well in obtaining the 3D CKT which preserves the symmetry between magnetic field and angular velocity. For this choice of momentum variables (7) is expressed as

$$
\Delta_{\mu}=\partial_{\mu}-\left[\Gamma_{\mu \lambda}^{\nu} p^{\lambda}+F_{\mu \lambda} g^{\lambda \nu}\right] \partial_{p \nu} .
$$

We would like to study (20) in the presence of the external electric and magnetic fields defined by $F_{0 i}=(\boldsymbol{E}-\boldsymbol{u} \times \boldsymbol{B})^{i}$ and $\frac{1}{2} \epsilon^{i j k} F_{j k}=-B^{i}$. Note that in a frame rotating with the angular velocity $\boldsymbol{\omega}$, Maxwell equations are given in terms of $\boldsymbol{B}$ and $\boldsymbol{E}^{\prime}=\boldsymbol{E}-\boldsymbol{u} \times \boldsymbol{B}$.

To derive the 3D CKT we would like to integrate (20) over $p^{0}$. To this aim we need to solve

$$
p^{2}+\hbar \frac{v_{\mu} \tilde{F}^{\mu \nu} p_{\nu}}{v \cdot p}=0
$$

for $p^{0}$ in terms of $p^{i}$. By adopting the notation of [27] let us introduce $\boldsymbol{q}=\left(p^{1}, p^{2}, p^{3}\right)$. The mass-shell condition can be solved as

$$
p^{0}= \pm \frac{|\boldsymbol{q}|}{\sqrt{g_{00}}} \sqrt{1+\frac{(\boldsymbol{q} \cdot \boldsymbol{u})^{2}}{\boldsymbol{q}^{2}}+\hbar \frac{F_{\mu \nu} S_{(v)}^{\mu \nu}}{\boldsymbol{q}^{2}}}-\frac{\boldsymbol{q} \cdot \boldsymbol{u}}{g_{00}} .
$$

Centrifugal terms are not taken into account, hence the rotation velocity satisfies (11). Moreover, we deal with weak external fields, so that we set $O\left(E^{2}\right) \approx 0$, and let the angular velocity and the magnetic field be in the same direction: $\boldsymbol{\omega} \times \boldsymbol{B}=0$. Therefore, in the semiclassical approximation, (21) yields

$$
p^{0}=\epsilon_{q}^{+}=|\boldsymbol{q}|-\boldsymbol{q} \cdot \boldsymbol{u}-\hbar|\boldsymbol{q}| \boldsymbol{b} \cdot \boldsymbol{B}^{\prime},
$$

where $\boldsymbol{b}=\boldsymbol{q} / 2|\boldsymbol{q}|^{3}$ is the Berry curvature and $\boldsymbol{B}^{\prime}=$ $\boldsymbol{B}-\boldsymbol{E} \times \boldsymbol{u}$ is the external magnetic field observed in the coordinate frame moving with the velocity $\boldsymbol{\omega} \times \boldsymbol{x}$, given by the Lorentz transformation with the Lorentz factor $\gamma=1 / \sqrt{1-\boldsymbol{u}^{2}} \approx 1$. Observe that this is the mass-shell condition which we should employ in performing $p^{0}$ integrals. As we will see the effective dispersion relation arising in the 3D CKT is independent of the electric field. We only exhibit the particle solution, although antiparticles are essential to perform momentum integrals. The positive part of the delta function becomes

$\delta^{+}\left(p^{2}+\hbar \frac{v_{\mu} \tilde{F}^{\mu \nu} p_{\nu}}{v \cdot p}\right)=\frac{\delta\left(p^{0}-\epsilon_{q}^{+}\right)}{2|\boldsymbol{q}|}[1-2 \hbar \boldsymbol{b} \cdot(\boldsymbol{E} \times \boldsymbol{u}-\boldsymbol{B})]$.

Now, by integrating (20) over $p^{0}$, one acquires

$$
\left.\left(\sqrt{\eta} \frac{\partial}{\partial t}+\sqrt{\eta} \dot{\boldsymbol{x}} \cdot \frac{\partial}{\partial \boldsymbol{x}}+\sqrt{\eta} \dot{\boldsymbol{q}} \cdot \frac{\partial}{\partial \boldsymbol{q}}+I_{0} \frac{\partial}{\partial p^{0}}\right) f\right|_{p^{0}=\epsilon_{q}^{+}}=0 .
$$

The phase space measure and the first time derivatives of phase space variables are

$$
\begin{aligned}
\sqrt{\eta}= & 1+\hbar \boldsymbol{b} \cdot(\boldsymbol{B}+2|\boldsymbol{q}| \boldsymbol{\omega})-\boldsymbol{\nu} \cdot \boldsymbol{u}, \\
\sqrt{\eta} \cdot \dot{\boldsymbol{x}}= & \boldsymbol{\nu}+\boldsymbol{E}^{\prime} \times \boldsymbol{b}+\hat{\boldsymbol{q}} \cdot \boldsymbol{b}(\boldsymbol{B}+2|\boldsymbol{q}| \boldsymbol{\omega})+2 \hat{\boldsymbol{q}} \boldsymbol{b} \cdot[\boldsymbol{u} \times \boldsymbol{E}], \\
\sqrt{\eta} \cdot \dot{\boldsymbol{q}}= & \boldsymbol{E}^{\prime}+\boldsymbol{\nu} \times(\boldsymbol{B}+2 \mathcal{E} \boldsymbol{\omega})+\hbar \boldsymbol{E} \cdot(\boldsymbol{B}+|\boldsymbol{q}| \boldsymbol{\omega}) \boldsymbol{b} \\
& +\hbar|\boldsymbol{q}| \boldsymbol{b} \cdot \boldsymbol{\omega} \boldsymbol{E}-[\boldsymbol{u} \times \boldsymbol{E}] \times \boldsymbol{\nu}+\hbar \hat{\boldsymbol{q}} \cdot \boldsymbol{b} \boldsymbol{E} \cdot \boldsymbol{B} \boldsymbol{u} \\
& +2 \boldsymbol{E} \cdot(\hat{\boldsymbol{q}} \times \boldsymbol{u}) \boldsymbol{b} \times \boldsymbol{B} .
\end{aligned}
$$

We introduced the "canonical velocity" $\nu=\partial \mathcal{E} / \partial \boldsymbol{q}$ with

$$
\mathcal{E}=q-\hbar q^{2}(\boldsymbol{b} \cdot \boldsymbol{\omega})-\hbar q(\boldsymbol{b} \cdot \boldsymbol{B}) .
$$

Observe that it is the semiclassical dispersion relation of a right-handed Weyl particle subject to the external electromagnetic fields in rotating coordinates [16]. When 
electromagnetic fields are present the coefficient of $\partial f / \partial p^{0}$ in (20) does not vanish. Its integral over $p^{0}$ turns out to be

$$
\begin{aligned}
I_{0}= & \hat{\boldsymbol{q}} \cdot \boldsymbol{E}^{\prime}(1+2 \hbar \boldsymbol{b} \cdot \boldsymbol{B}+\hbar|\boldsymbol{q}| \boldsymbol{b} \cdot \boldsymbol{\omega})+\hbar \boldsymbol{b} \cdot \boldsymbol{q} \boldsymbol{E} \cdot \boldsymbol{\omega} \\
& -\boldsymbol{E} \cdot \boldsymbol{u}-(1+2 \hbar \boldsymbol{b} \cdot \boldsymbol{B}) \hat{\boldsymbol{q}} \cdot(\boldsymbol{B} \times \boldsymbol{u})-\hbar \boldsymbol{b} \cdot \boldsymbol{u} \boldsymbol{E} \cdot \boldsymbol{B} .
\end{aligned}
$$

However, one can show that it can be expressed as

$$
I_{0}=\sqrt{\eta} \dot{\boldsymbol{q}} \cdot\left(\partial \epsilon_{q}^{+} / \partial \boldsymbol{q}\right)
$$

Hence, when we integrate (20) over $p^{0}$, the coefficients of $\partial f / \partial p^{\mu}$, namely the last two terms of (23), lead to

$$
\begin{aligned}
& \sqrt{\eta} \dot{\boldsymbol{q}} \cdot\left[\frac{\partial f(x, p)}{\partial \boldsymbol{q}}\right]_{p^{0}=\epsilon_{q}^{+}}+\sqrt{\eta} \dot{\boldsymbol{q}} \cdot \frac{\partial \epsilon_{q}^{+}}{\partial \boldsymbol{q}}\left[\frac{\partial f(x, q)}{\partial p^{0}}\right]_{p^{0}=\epsilon_{q}^{+}} \\
& =\sqrt{\eta} \dot{\boldsymbol{q}} \frac{\partial f\left(t, \boldsymbol{x}, \epsilon_{q}^{+}, \boldsymbol{q}\right)}{\partial \boldsymbol{q}} .
\end{aligned}
$$

Therefore, we establish the 3D CTE as

$$
\left(\sqrt{\eta} \frac{\partial}{\partial t}+\sqrt{\eta} \dot{\boldsymbol{x}} \cdot \frac{\partial}{\partial \boldsymbol{x}}+\sqrt{\eta} \dot{\boldsymbol{q}} \cdot \frac{\partial}{\partial \boldsymbol{q}}\right) f(t, \boldsymbol{x}, \boldsymbol{q})=0,
$$

where $f(t, \boldsymbol{x}, \boldsymbol{q}) \equiv f\left(t, \boldsymbol{x}, p^{0}=\epsilon_{q}^{+}, \boldsymbol{q}\right)$.

By making use of (24)-(26) and the Maxwell equations in rotating coordinates one can show that the Liouville equation satisfied by the measure is

$$
\frac{\partial}{\partial t} \sqrt{\eta}+\frac{\partial}{\partial \boldsymbol{x}}(\sqrt{\eta} \dot{\boldsymbol{x}})+\frac{\partial}{\partial \boldsymbol{q}}(\sqrt{\eta} \dot{\boldsymbol{q}})=(2 \pi \delta(\boldsymbol{q})+2 \hat{\boldsymbol{b}} \cdot \boldsymbol{u}) \boldsymbol{E} \cdot \boldsymbol{B} .
$$

In 3D the chiral particle number and current densities are defined as

$$
\begin{aligned}
& n=\int[d q] \sqrt{\eta} f, \\
& \boldsymbol{j}=\int[d q] \sqrt{\eta} \cdot \dot{\boldsymbol{x}} f+\boldsymbol{j}_{M},
\end{aligned}
$$

where $[d q]=d^{3} q /(2 \pi \hbar)^{3}$ and

$$
\boldsymbol{j}_{M}=\boldsymbol{\nabla} \times \int[d q] \hbar \mathcal{E} \boldsymbol{b} f
$$

is the magnetization current $[14,17,33,34]$.

By making use of (27) and (28), one can show that the 4-divergence of the 4-current $(n, \boldsymbol{j})$ yields the continuity equation with source:

$$
\frac{\partial n}{\partial t}+\boldsymbol{\nabla} \cdot \boldsymbol{j}=\left.\frac{\boldsymbol{E} \cdot \boldsymbol{B}}{(2 \pi \hbar)^{2}} f\right|_{\boldsymbol{q}=0}
$$

Note that on the right-hand side only $f^{0}$ appears. This continuity equation is consistent with the chiral anomaly.

Let us now focus on the currents which are proportional to $\boldsymbol{B}$ and $\boldsymbol{\omega}$, by setting $\boldsymbol{E}=0$. For $p^{0}=\epsilon_{q}^{+}$, the equilibrium distribution function (14) with $n_{\mu}=U_{\mu}=v_{\mu}$, becomes

$$
f(t, \boldsymbol{x}, \boldsymbol{p})=\frac{1}{e^{(\mathcal{E}-\mu) / T}+1} .
$$

By plugging it into (30) and employing (25) one attains the chiral magnetic and vortical effects correctly:

$$
\boldsymbol{j}=\frac{\left(3 \mu^{2}+T^{2} \pi^{2}\right)}{12 \pi^{2} \hbar^{2}} \boldsymbol{\omega}+\frac{\mu}{4 \pi^{2} \hbar^{2}} \boldsymbol{B} .
$$

To calculate the integrals we have taken into account both particle and antiparticle contributions. We conclude that (24)-(27) describe a consistent 3D CKT.

\section{DISCUSSIONS}

In Minkowski spacetime the modified chiral QKE generates a consistent 3D CKT which does not explicitly depend on spatial coordinates. In Sec. III we studied its curved spacetime formulation by ignoring centrifugal force terms imposing the conditions (11). The modification terms survive for the inertial observer: $n_{\mu}=u_{\mu}$. We derived the current density at $\boldsymbol{x} \approx 0$ and show that the chiral vortical effect is generated for the particle (fluid) velocity $U_{\mu}=v_{\mu}$. For $U_{\mu}=u_{\mu}$ the current density does not lead to any angular momentum dependent term. This is not surprising because the equilibrium distribution function of rotating fluid in Minkowski spacetime [31] is consistent only with the former choice. We derived the transport equation in the absence of electromagnetic fields as in (16). It furnishes the phase space measure and velocities which in part coincide with the ones obtained in [16] at $\boldsymbol{x} \approx 0$. Hence, we can conclude that the modified QKE in curved spacetime is needed to acquire the phase space measure and the first time derivatives of phase space variables correctly for the inertial observer.

In Sec. IV the novel CTE (27) is established for $n_{\mu}=v_{\mu}$. It is similar to the CKT obtained directly in 3D [16]. The difference is mainly in the explicitly $\boldsymbol{x}$-dependent terms of (24)-(26). The 3D CKT of [16] was constructed starting from the scalar and vector fields which can be associated with Coriolis and centrifugal forces experienced by a massive particle. One can examine if a similar approach in 3D exists which can be associated with the new 3D CKT. Another open question is how to incorporate the centrifugal force in the 3D CKT starting with the 4D curved spacetime formulation of chiral particles. It is a hard task, because one should not only keep terms at the order of $\boldsymbol{u}^{2}$ but also, at least initially, the $\boldsymbol{u}^{3}$ terms whose derivatives with respect to spatial coordinates are at the order of $\boldsymbol{u}^{2}$. This would also clarify if an underlying 3D construction mentioned above exists. 
We only presented transport equations without collisions. The explicitly $\boldsymbol{x}$-dependent CKT of [16] has been extended to cover collisions by adopting the relaxation time method [35]. This allowed us to study nonlinear transport properties of chiral plasma. The novel formalism constructed here can be studied in a similar manner. Collisions can be introduced by means of the relaxation time formalism and the particle current densities provided by them can be calculated. Once this is done one can compare the particle currents generated by these CKTs. This would serve as a testing ground for deciding which CKT suits better with the observable effects.

\section{ACKNOWLEDGMENTS}

We would like to thank Xu-Guang Huang for the illuminative correspondence on their work. This work is supported by the Scientific and Technological Research Council of Turkey (TÜBITAK) Grant No. 117 F328.
[1] D. E. Kharzeev, L. D. McLerran, and H. J. Warringa, The effects of topological charge change in heavy ion collisions: Event by event P and CP violation, Nucl. Phys. A803, 227 (2008).

[2] K. Fukushima, D. E. Kharzeev, and H. J. Warringa, Chiral magnetic effect, Phys. Rev. D 78, 074033 (2008).

[3] D. Kharzeev and A. Zhitnitsky, Charge separation induced by P-odd bubbles in QCD matter, Nucl. Phys. A797, 67 (2007).

[4] M. A. Metlitski and A. R. Zhitnitsky, Anomalous axion interactions and topological currents in dense matter, Phys. Rev. D 72, 045011 (2005).

[5] K. Jensen, P. Kovtun, and A. Ritz, Chiral conductivities and effective field theory, J. High Energy Phys. 10 (2013) 186.

[6] A. Vilenkin, Quantum field theory at finite temperature in a rotating system, Phys. Rev. D 21, 2260 (1980).

[7] D. T. Son and P. Surówka, Hydrodynamics with Triangle Anomalies, Phys. Rev. Lett. 103, 191601 (2009).

[8] A. V. Sadofyev, V. I. Shevchenko, and V. I. Zakharov, Notes on chiral hydrodynamics within the effective theory approach, Phys. Rev. D 83, 105025 (2011).

[9] N. Banerjee, J. Bhattacharya, S. Bhattacharyya, S. Dutta, R. Loganayagam, and P. Surówka, Hydrodynamics from charged black branes, J. High Energy Phys. 01 (2011) 094.

[10] K. Landsteiner, E. Megias, and F. Pena-Benitez, Gravitational Anomaly and Transport Phenomena, Phys. Rev. Lett. 107, 021601 (2011).

[11] Z. T. Liang and X. N. Wang, Globally Polarized QuarkGluon Plasma in Noncentral A + A Collisions, Phys. Rev. Lett. 94, 102301 (2005).

[12] F. Becattini, F. Piccinini, and J. Rizzo, Angular momentum conservation in heavy ion collisions at very high energy, Phys. Rev. C 77, 024906 (2008).

[13] J.-H. Gao, Z.-T. Liang, S. Pu, Q. Wang, and X-N. Wang, Chiral Anomaly and Local Polarization Effect from the Quantum Kinetic Approach, Phys. Rev. Lett. 109, 232301 (2012).

[14] D. T. Son and N. Yamamoto, Kinetic theory with Berry curvature from quantum field theories, Phys. Rev. D 87, 085016 (2013).

[15] M. A. Stephanov and Y. Yin, Chiral Kinetic Theory, Phys. Rev. Lett. 109, 162001 (2012).
[16] Ö. F. Dayi, E. Kilinçarslan, and E. Yunt, Semiclassical dynamics of Dirac and Weyl particles in rotating coordinates, Phys. Rev. D 95, 085005 (2017).

[17] C. Y. Hidaka, S. Pu, and D. L. Yang, Relativistic Chiral kinetic theory from quantum field theories, Phys. Rev. D 95, 091901 (2017).

[18] C. Y. Hidaka, S. Pu, and D. L. Yang, Nonlinear responses of chiral fluids from kinetic theory, Phys. Rev. D 97, 016004 (2018).

[19] A. Huang, S. Shi, Y. Jiang, J. Liao, and P. Zhuang, Complete and consistent chiral transport from Wigner function formalism, Phys. Rev. D 98, 036010 (2018).

[20] H. T. Elze, M. Gyulassy, and D. Vasak, Transport equations for the QCD quark Wigner operator, Nucl. Phys. B276, 706 (1986).

[21] D. Vasak, M. Gyulassy, and H. T. Elze, Quantum transport theory for Abelian plasmas, Ann. Phys. (N.Y.) 173, 462 (1987).

[22] Ö. F.Dayi and E. Kilinçarslan, Quantum kinetic equation in the rotating frame and chiral kinetic theory, Phys. Rev. D 98, 081701(R) (2018).

[23] J.-W. Chen, S. Pu, Q. Wang, and X.-N. Wang, Berry Curvature and Four-Dimensional Monopoles in the Relativistic Chiral Kinetic Equation, Phys. Rev. Lett. 110, 262301 (2013).

[24] J.-H. Gao, S. Pu, and Q. Wang, Covariant chiral kinetic equation in Wigner function approach, Phys. Rev. D 96, 016002 (2017).

[25] N. Mueller and R. Venugopalan, Worldline construction of a covariant chiral kinetic theory, Phys. Rev. D 96, 016023 (2017).

[26] S. Carignano, C. Manuel, and J. M. Torres-Rincon, Consistent relativistic chiral kinetic theory: A derivation from on-shell effective field theory, Phys. Rev. D 98, 076005 (2018).

[27] Y.-C. Liu, L.-L. Gao, K. Mameda, and X.-G. Huang, Chiral kinetic theory in curved spacetime, Phys. Rev. D 99, 085014 (2019).

[28] O. A. Fonarev, Wigner function and quantum kinetic theory in curved spacetime and external fields, J. Math. Phys. (N.Y.) 35, 2105 (1994).

[29] C. W. Misner, K. S. Thorne, and J. A. Wheeler, Gravitation (Freeman, San Francisco, 1973). 
[30] F. W. Hehl and W.-T. Ni, Inertial effects of a Dirac particle, Phys. Rev. D 42, 2045 (1990).

[31] B. J.-Y. Chen, D. T. Son, and M. A. Stephanov, Collisions in Chiral Kinetic Theory, Phys. Rev. Lett. 115, 021601 (2015).

[32] X.-G. Huang and A. V. Sadofyev, Chiral vortical effect for an arbitrary spin, J. High Energy Phys. 03 (2019) 084.
[33] A. J.-Y. Chen, D. T. Son, M. A. Stephanov, H.-U. Yee, and Y. Yin, Lorentz Invariance in Chiral Kinetic Theory, Phys. Rev. Lett. 113, 182302 (2014).

[34] J.-W. Chen, T. Ishii, S. Pu, and N. Yamamoto, Nonlinear chiral transport phenomena, Phys. Rev. D 93, 125023 (2016).

[35] Ö. F. Dayi and E. Kilinçarslan, Nonlinear chiral plasma transport in rotating coordinates, Phys. Rev. D 96, 043514 (2017). 